\title{
ENERGY RESOLUTION ENHANCEMENT OF MERCURIC IODIDE DETECTORS
}

\author{
M. Finger and T. A. Prince \\ California Institute of Technology, Pasadena, CA 91125 \\ L. Padgett \\ Santa Barbara Research Center, Goleta, CA 93117 \\ B. Prickett \\ Xicor Inc., Milpitas, CA 95035 \\ W. Schnepple \\ EG\&G, Santa Barbara Operations, Goleta, CA 93117
}

\begin{abstract}
A pulse processing technique has been developed which improves the gamma-ray energy resolution of mercuric iodide detectors. The technique employs a fast (100 ns) and a slow $(6.4 \mu \mathrm{s})$ pulse height analysis to correct for signal variations due to variations in charge trapping. The capabilities of the technique for energy resolution enhancement are discussed as well as the utility of the technique for examining the trapping characteristics of individual detectors. We have achieved an energy resolution of $2.6 \%$ FWHM at $662 \mathrm{keV}$ with an acceptance efficiency of $100 \%$ from a mercuric iodide detector which gives $8.3 \%$ FWHM using standard techniques.
\end{abstract}

\section{Introduction}

Many studies of $x$-ray and $\gamma$-ray lines demand high resolution detectors for the precise determination of line energies. Although silicon and germanium detectors have excellent energy resolution, the need for refrigeration often makes their application difficult or even prohibitive. It would thus be beneficial to develop detectors having good energy resolution that can also be operated at room $\left(30^{\circ} \mathrm{C}\right)$ temperature.

The search for suitable $\gamma$-ray detectors has led to the development of two high-Z room temperature solid state detector materials, mercuric iodide $\left(\mathrm{HgI}_{2}\right)$ and cadmium telluride (CdTe) (c.f. the review by Whited and Schieber ${ }^{1}$ ). Although considerable progress has been made in the utilization of these materials during the past ten years, there has been a persistent difficulty in obtaining good energy resolution. In $\mathrm{HgI}_{2}$ detectors the energy resolution is limited by excessive charge trapping coupled with low hole mobility.

Techniques have been developed to overcome some of the resolution problems in low-energy $\mathrm{x}$-ray spectroscopy ${ }^{2}$. These applications take advantage of the small $x$-ray penetration depth at low energy. With $x$-rays incident on the negative electrode of the detector, the signal is predominantly due to electron carriers and this effectively eliminates the problem of hole transport and trapping. Unfortunately, as the photon energy increases the penetration depth also increases and such techniques lose their effectiveness. At $662 \mathrm{keV}$ for example, typical detectors have resolutions of $\geq 10 \%$, although a few exceptional detectors have been produced. ${ }^{1}$

We present in this paper an electronic technique for enhancing the energy resolution of $\mathrm{HgI}_{2}$ detectors and other similar semiconductor detectors. The basic principle of the technique is straightforward: in addition to the total signal in the detector, we also measure the relative contribution of hole and electron carriers and use this information to correct for the reduction in total signal due to hole trapping. In general, the larger the contribution of the hole carriers, the greater the correction to the measured total signal. In $\mathrm{HgI}_{2}$, the mobilities for electron and hole carriers differ by a factor of $25\left(\sim 100 \mathrm{~cm}^{2} /\left(\mathrm{V}^{*} \mathrm{~s}\right)\right.$ for electrons and $\sim 4 \mathrm{~cm}^{2} /\left(\mathrm{V}^{*} \mathrm{~s}\right)$ for holes). We may take advantage of this fact to measure the relative contributions of hole and electron carriers by analysis of the signal at two different times: a fast measurement at $\sim 100 \mathrm{~ns}$ which responds primarily to the electron component of the signal, and a slow time constant measurement $(6.4 \mu \mathrm{s})$ which is proportional to the total contribution of both hole and electron carriers.
Other electronic techniques which have been tried in the past to enhance the energy resolution of $\mathrm{HgI}_{2}$ and $\mathrm{CdTe}$ detectors include rise time discrimination ${ }^{3,4}$ and ballistic deficit corrections. ${ }^{5}$ Rise time discrimination selects those events in which the signal is dominated by electron carriers. While improved energy resolution is obtained, the efficiency at high energies is quite low since only a small fraction of the events deposit their full energy very near the negative electrode. Our method relies on a fast time constant measurement of the charge signal rather than a rate of rise measurement and attains $100 \%$ acceptance efficiency.

\section{Detector Operation and Electronics}

Figure 1 shows a simplified block diagram of the charge collection in a $\mathrm{HgI}_{2}$ detector. A simple model provides a quantitative understanding of the detector response. Let us assume that the bias voltage $V$ creates a uniform electric field across the thickness $d$ of the detector. As in Figure 1, a $\gamma$-ray interacts in the detector forming a cloud of $\mathrm{N}$ electron-hole pairs localized a distance $z$ from the positive electrode. The bias field causes the electrons to move toward the positive electrode and the holes to move toward the negative electrode with velocities,

$$
v_{\mathrm{e}}=\mu_{\mathrm{e}} V / d, \text { and } v_{h}=\mu_{\mathrm{h}} V / d \text {, }
$$

respectively, where $\mu_{\mathrm{e}}$ is the electron mobility and $\mu_{\mathrm{h}}$ is the hole mobility. The electrons and holes arrive at the respective electrodes at times,

$$
t_{e}=z / v_{e}, \text { and } t_{h}=(\mathrm{d}-\mathrm{z}) / v_{h}
$$

The maximum transit times for electrons and holes are $T_{\theta}=d / v_{s}$ and $T_{h}=d / v_{h}$ respectively. For a $500 \mu \mathrm{m}$ thick detector and an applied voltage of $1000 \mathrm{~V}$, the maximum transit time is $\sim 25 \mathrm{~ns}$ for electrons and $\sim 0.6 \mu$ s for holes.

The current $I_{D E T}$ in the external circuit is:

$$
I_{D E T}=i_{\varepsilon}+i_{h} \equiv N q v_{e} v_{e}(t) / d+N q v_{h} v_{h}(t) / d .
$$

where: $q=$ charge of the electron,

$\vartheta_{e}=1$ for $\left\{0<t<t_{e}\right\} ; v_{e}=0$ otherwise, and

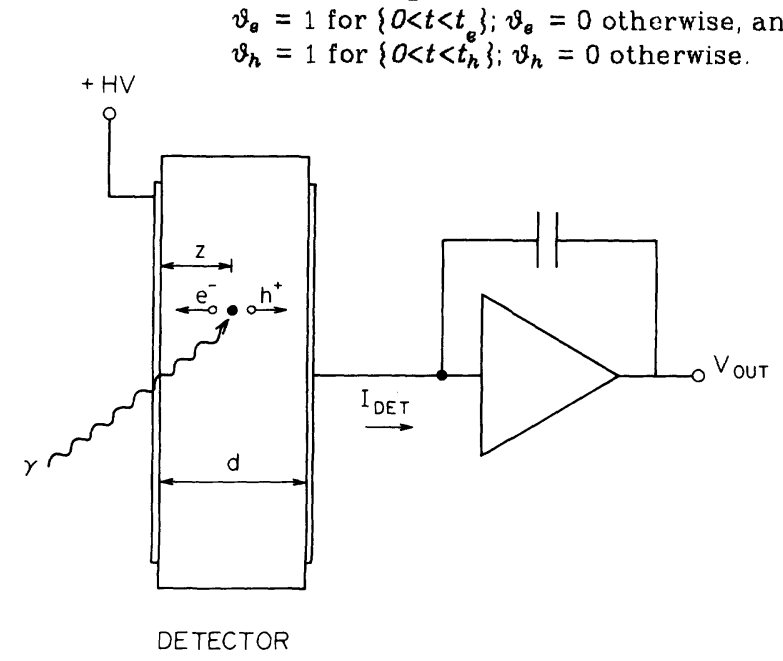

Figure 1: Schematic of detector operation. 


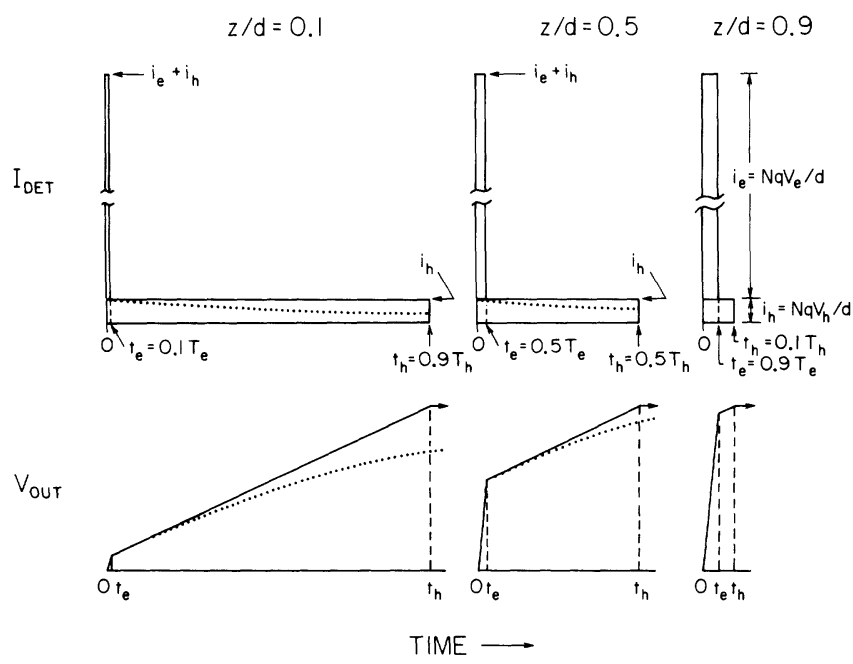

Figure 2: Detector current pulse and preamp voltage output. Notation is defined in the text.

Figure 2 illustrates the current pulses produced in the detector by a point interaction at several depths in the detector and the corresponding response ( $V_{\text {OUT }}$ ) of a perfect charge integrating amplifier. Note that the amplitude of the electron or hole component of the current depends only on the number of charge pairs, while the duration of each component is controlled by the initial location of the charge cloud. Since the electron velocity is much larger then the hole velocity the integrated current or "collected charge" rises abruptly to a fraction $z / d$ of the total collected charge $N q$. Thus we see that the signal shape contains information about the location of the event in the detector.

So far we have ignored the effects of hole trapping. As the holes travel toward the negative electrode their number will decrease exponentially resulting in a deficit in the total collected charge. This deficit depends on the distance the holes must travel within the detector and so varies with the interaction depth. To include trapping, the function $\vartheta_{h}$ in equation ( 3 ) must be modified:

$$
v_{h}=e^{\frac{-t}{\tau_{h}}} \text { for }\left\{0<t<t_{h}\right\}
$$

where $\tau_{h}$ is the characteristic hole trapping time. The dotted curves in Figure 2 shows the effect of trapping on the current signal and its integral. For times greater than $t_{h}, \vartheta_{h}$ may not be exactly zero due to subsequent release of hole carriers from shallow traps ("detrapping"). In fact, it is a standard practice to use signal integration times considerably longer than $0.5 \mu$ s in order to collect this "late" charge.

The two dimensional pulse processing technique involves a measurement of the integral charge at times of $100 \mathrm{~ns}$ and
6.4 $\mu \mathrm{s}$, which we will call $\mathrm{S}_{\text {fast }}$ and $\mathrm{S}_{\text {slow }}$ respectively. By using the ratio of $S_{\text {fast }}$ to $S_{\text {slow }}$ the ratio of electron carriers to hole carriers can be estimated. The average position of ionization deposition in the detector can then be determined and the effects of trapping can be corrected in an empirical manner.

Note that the two dimensional pulse processing technique is sensitive to the energy weighted interaction position. A photon may exhibit a multiple point interaction, for instance a photoelectric conversion followed by an x-ray fluorescence conversion. In the approximation that trapping simply depends on the product of the number of hole carriers times the distance through which they travel, this complication should not affect the basic two dimensional pulse processing technique.

\section{Two Dimensional Pulse Processing Electronics}

Figure 3 shows schematically the electronic processing used in the analysis. The current pulse from the $\mathrm{HgI}_{2}$ detector was amplified by a fast charge sensitive preamplifier and split into two pulse processing channels: a slow channel with a $6.4 \mu \mathrm{s}$ time constant, and a fast channel with a sampling time of $100 \mathrm{~ns}$.

The fast preamplifier was a Tennelec TC161D direct coupled charge sensitive preamplifier with a rise time of $35 \mathrm{~ns}$. The detector, preamplifier, and HV filter were enclosed in a light tight rf shielded box.

The slow channel utilized a Tennelec TC200 variable time constant amplifier (integration and differentiation time constants set to $6.4 \mu \mathrm{s}$ ) to produce a signal whose amplitude was proportional to the total charge collected from the detector. The output was fed to a multiple parameter pulse height analyzer system which digitized the signal, together with the fast channel output, and recorded it on tape for subsequent analysis.

The fast pulse processing channel was configured to produce a signal approximating the magnitude of the collected charge due to electron carriers. The preamplifier signal was buffered and split into two branches. One branch went to an EG\&G LG102 integrating linear gate, which produced an output signal whose amplitude was proportional to the integral of the input signal during a gate interval. The other branch, consisting of an EG\&G TD101 discriminator plus associated delay lines, produced the gate interval signal for the linear gate. The gate interval signal was delayed so that the sampling interval avoided the input signal rise time. The gate interval width was set by an empirical tradeoff between high frequency noise in the input signal and contamination by the hole component of the signal.

Figure 4 shows the characteristic signal shape and processing times associated with the fast pulse processing channel. The electronics were configured to produce a signal which was a measure of the "collected charge" at a time (100 $\mathrm{ns}$ ) shortly after the maximum transit time of electron

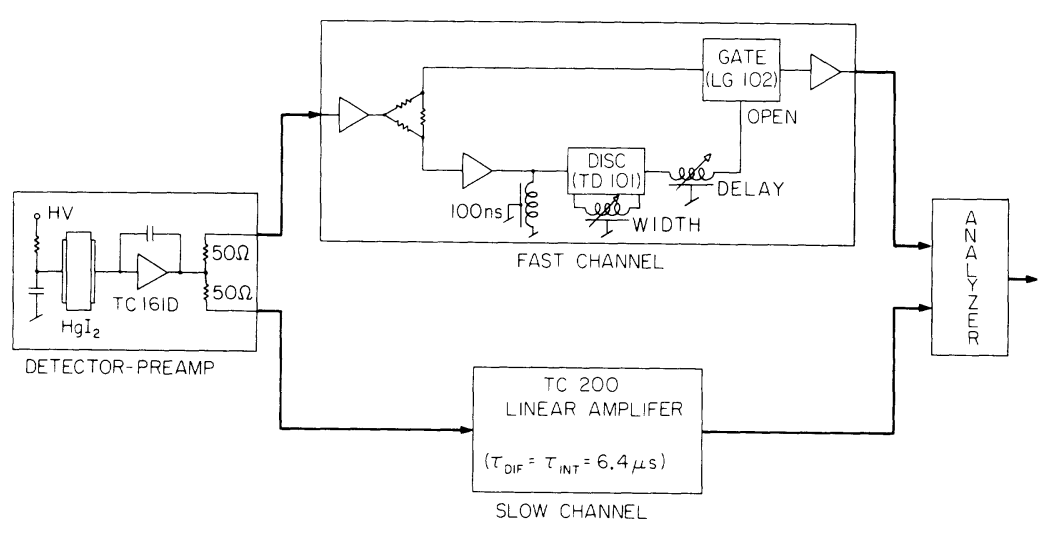

Figure 3: Schematic diagram of the electronics.

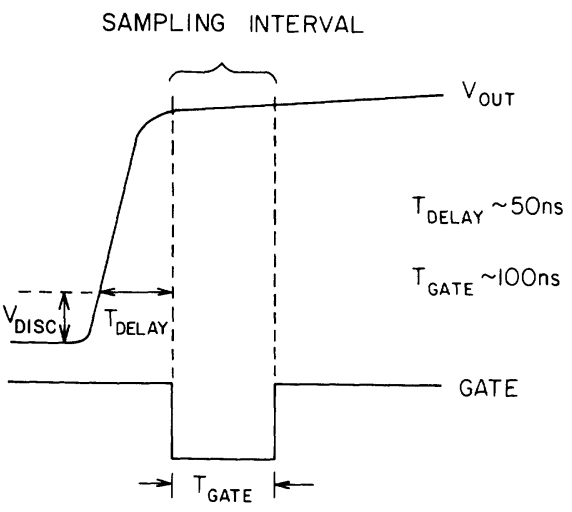

Figure 4: Operation of the fast pulse processor. $V_{D I S C}$ is the discriminator threshold. 
carriers in the detector. The measured signal was predominantly due to electron carriers with a small admixture of hole carrier signal.

\section{Results}

After examining a number of detectors, it became clear that different detectors exhibited qualitatively different slow versus fast signal characteristics, indicating substantial differences in both the degree and nature of energy degradation effects such as charge trapping. We present the results from three detectors to indicate the possible range of behaviors.

The detectors used in this study were fabricated at EG\&G Santa Barbara from crystal material purified and grown on site. All detectors had vacuum deposited palladium electrodes. The table lists the characteristics of the detectors reported on here.

\section{Fnergy Resolution Bnhancement}

Figure $5 \mathrm{a}$ shows the event scatter plot distribution produced by $662 \mathrm{keV}{ }^{137} \mathrm{Cs} \gamma$-rays in detector S8-13. Of all detectors studied, this detector exhibited the best nominal energy resolution at both 59.5 and $662 \mathrm{keV}$. The events in

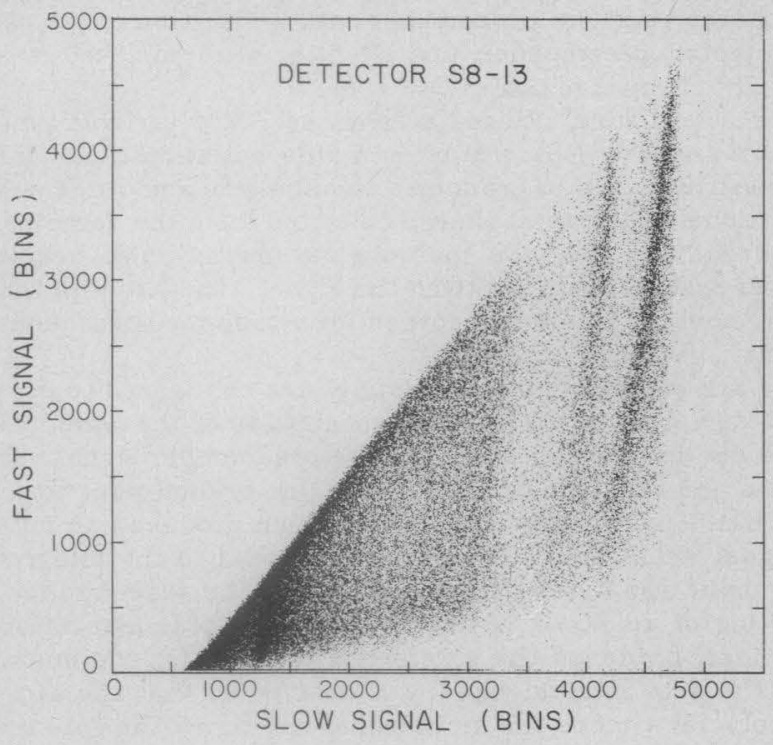

Figure 5a: Fast versus slow signal distribution.

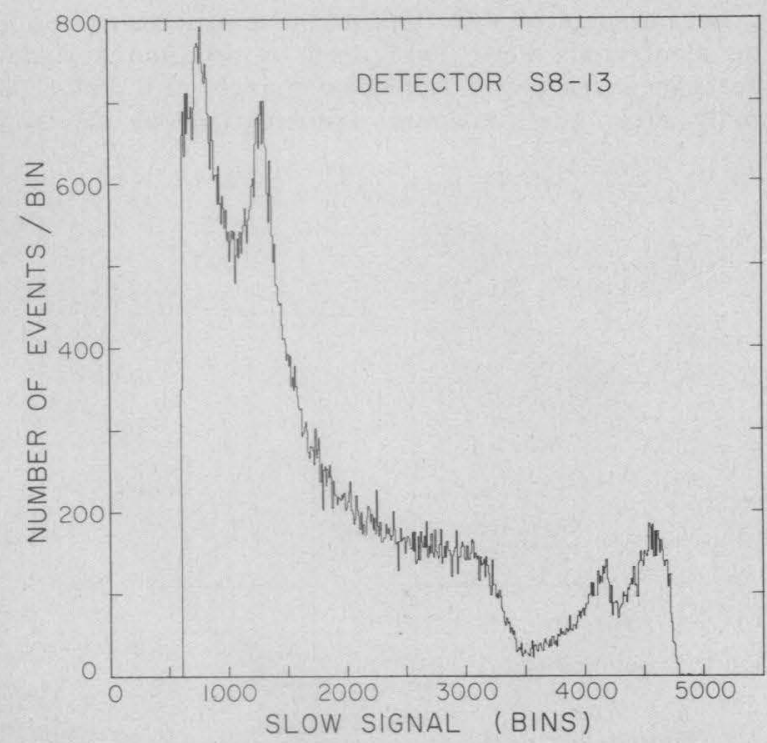

Figure 5b: Histogram of uncorrected energy loss.

\begin{tabular}{|l||c|c|cr|}
\hline Detector \# & $\begin{array}{c}\text { Area } \\
\left(\mathrm{cm}^{2}\right)\end{array}$ & $\begin{array}{c}\text { Thickness } \\
(\mu \mathrm{m})\end{array}$ & \multicolumn{2}{|c|}{$\begin{array}{c}\text { Energy Resolution } \\
\text { (FWHM) }\end{array}$} \\
& & & $59.5 \mathrm{keV}$ & $662 \mathrm{keV}$ \\
\hline S8-13 (TM1) & .73 & 310 & $5.7 \%$ & $8.3 \%$ \\
S10-3 (LP53) & .66 & 536 & $7.4 \%$ & $\sim 12 \%$ \\
S6-25 (LP51) & .65 & 535 & $6.4 \%$ & $>20 \%$ \\
\hline
\end{tabular}

Figure $5 \mathrm{a}$ are located in a triangular slice of the fast versus slow signal plane. The upper boundary corresponds to the case of exclusive electron carrier transport, while the lower boundary corresponds to the case of exclusive hole carrier transport. The intervening area on the plot corresponds to varying average interaction depths of the events in the detector.

A prominent "track" is present in the right hand portion of the plot beginning at the upper boundary and trailing off towards smaller fast and slow signals, accompanied by broadening and possible curvature of the track. This feature is due to the $662 \mathrm{keV}$ full energy events. Next to the prominent full energy track is the less intense escape track at 579 $\mathrm{keV}$ due to events in which a $83 \mathrm{keV}(\mathrm{Hg} \mathrm{K}) \mathrm{x}$-ray escapes from the detector.



Figure 5c: Corrected event distribution showing estimated interaction depth versus estimated energy loss.

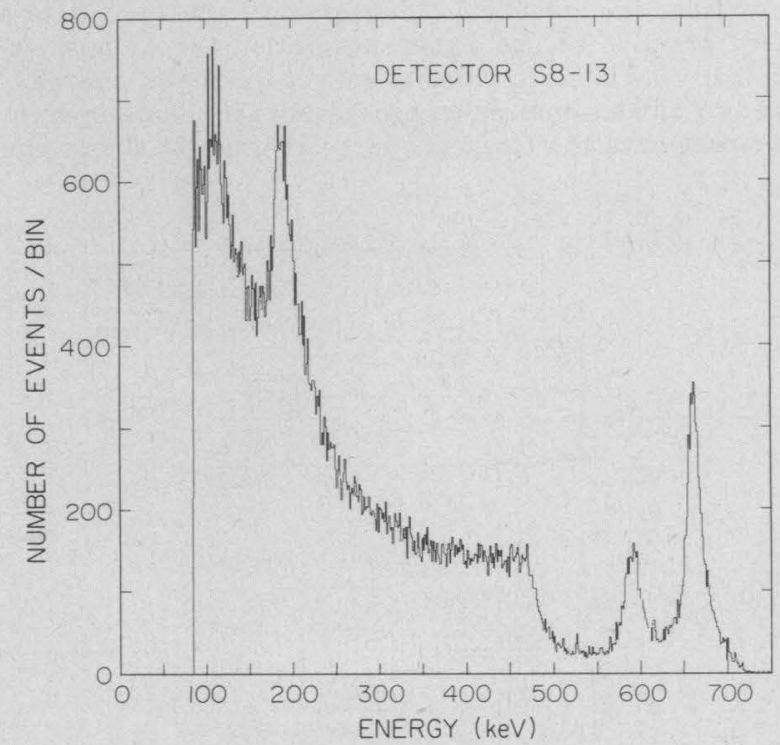

Figure 5d: Histogram of corrected energy loss. 
With standard one dimensional pulse processing techniques, only the slow signal is measured and the resulting "energy loss" distribution is shown in Figure 5b. Significant energy resolution degradation occurs with the standard analysis because there is no ability to correct for the dependence of the signal on the depth of interaction. This dependence evidences itself as the curvature and slope of the energy loss tracks in the fast versus slow signal plot.

Figures $5 c$ and $5 d$ show the energy resolution enhancement derived from the fast versus slow signal plot. Figure $5 \mathrm{c}$ shows the estimated depth of interaction versus the estimated energy loss of the events. The estimated energy loss was obtained from the event distribution of Figure 5a by an empirical correction for the slope and curvature of the energy loss tracks. Figure $5 \mathrm{~d}$ shows the resulting energy loss histogram. Comparison of Figure $5 b$ and Figure $5 d$ indicates a significant enhancement of the energy resolution. The energy resolution improves from 8.3\% FWHM to $2.6 \%$ FWHM at 662 $\mathrm{keV}$ and the peak to valley ratio for the full energy peak improves from $2: 1$ to $9: 1$. This energy resolution enhancement was obtained with no loss of efficiency. It is obvious from the plots that even better resolution can be obtained by restricting the event selection to the upper portion of the energy loss tracks, albeit with a corresponding loss in efficiency.

\section{Model Calculations}

The two dimensional pulse processing method is obviously a useful empirical technique for enhancing the energy resolution of detectors such as $\mathrm{HgI}_{2}$. However, a quantitative understanding of the distribution of events in the fast versus slow signal plane can lead to a better understanding of the sources of energy degradation of individual detectors. We have therefore expanded the model for the detector operation given in Section II to include the operation of the electronics. The model thus predicts both the time dependence of the current signal from the detector and the resulting fast and slow output signals.

The model assumes a uniform electric field, uniform density of hole traps, negligible electron trapping, and equal initial numbers of electron and hole carriers. Detrapping of the hole carriers is neglected. Electronic characteristics such as gate integration time, discriminator gate time jitter, and ballistic deficit in the slow signal measurement were explicitly included.

Three parameters describe the charge transport in the model: the electron and hole mobilities and the hole trapping time. The electron and hole mobilities were taken to be 100 $\mathrm{cm}^{2} /\left(\mathrm{V}^{*} \mathrm{~s}\right)$ and $4 \mathrm{~cm}^{2} /\left(\mathrm{V}^{*} \mathrm{~s}\right)$ respectively. The hole trapping time was a free parameter of the the model.

Explicitly, the model predicts the following amplitudes for the slow and fast signals:

$$
\begin{aligned}
& S_{\text {slow }}=N q\left\{1-\frac{\bar{z}}{d}\left[1+\frac{\bar{\tau}}{t_{h}}\left[1-e^{\frac{t_{h}}{\bar{\tau}}}\left(1-\left(\left(t_{h}-\bar{\tau}\right) / \tau_{R C}\right)\right)+\frac{\bar{\tau}}{\tau_{R C}}\right)\right]\right\},(4 \mathrm{a}) \\
& S_{\text {fast }}=N q\left\{1-\frac{\bar{z}}{d}\left[1-\frac{\tau_{h}}{t_{h}}\left(1-e^{\frac{-T_{0}}{\tau_{h}}} \frac{\tau_{h}}{T_{G A T E}}\left(1-e^{\frac{-T_{G A T E}}{T_{h}}}\right)\right)\right]\right\} . \\
& \text { where: } \overline{\boldsymbol{z}}=\boldsymbol{d}-\boldsymbol{z} \text {, } \\
& \bar{T}=\left(1 / \tau_{R C}-1 / \tau_{h}\right)^{-1} . \\
& \tau_{R C}=\mathrm{CR}-\mathrm{RC} \text { shaping time }=6.4 \mu \mathrm{s} \text {. } \\
& T_{0}=\text { start time of integration }=50 \mathrm{~ns} \text {, and } \\
& T_{\text {CATE }}=\text { gate } \text { width }=100 \mathrm{~ns} \text {. }
\end{aligned}
$$

In Figures $6 \mathrm{a}-\mathrm{c}$ we present results for detector $\mathrm{S} 10-3$ similar to those presented in Figures $5 a, 5 b$, and $5 d$ for detector SB-13. Detector S10-3 exhibited considerably worse energy resolution than detector S8-13, particularly at high $\gamma$-ray energies such as $662 \mathrm{keV}$ (c.f. Figures $5 \mathrm{~b}$ and $6 \mathrm{~b}$ ). Figure $6 a$ shows the results of model calculations for several



Figure 6a: Fast versus slow signal distribution.

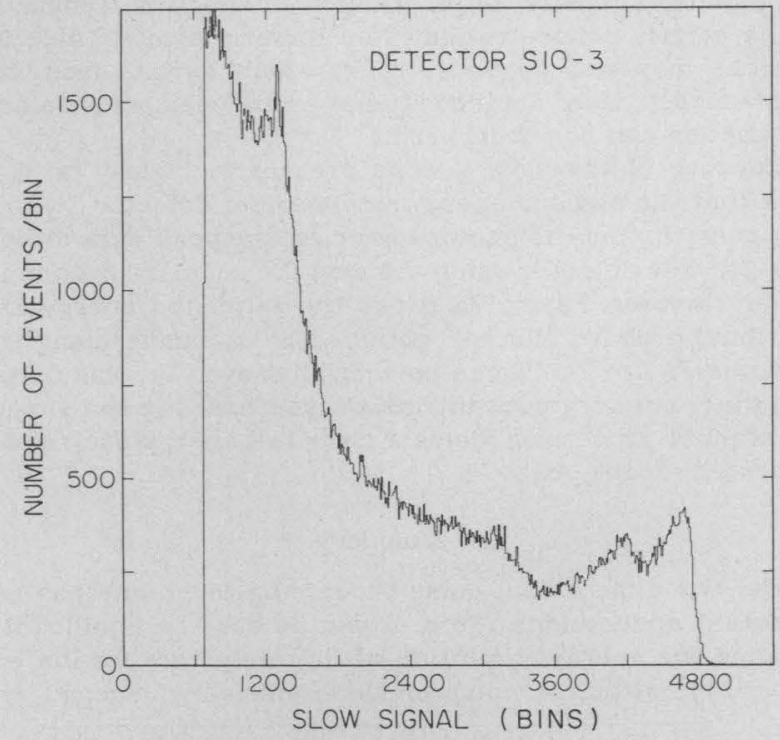

Figure 6b: Histogram of uncorrected energy loss.

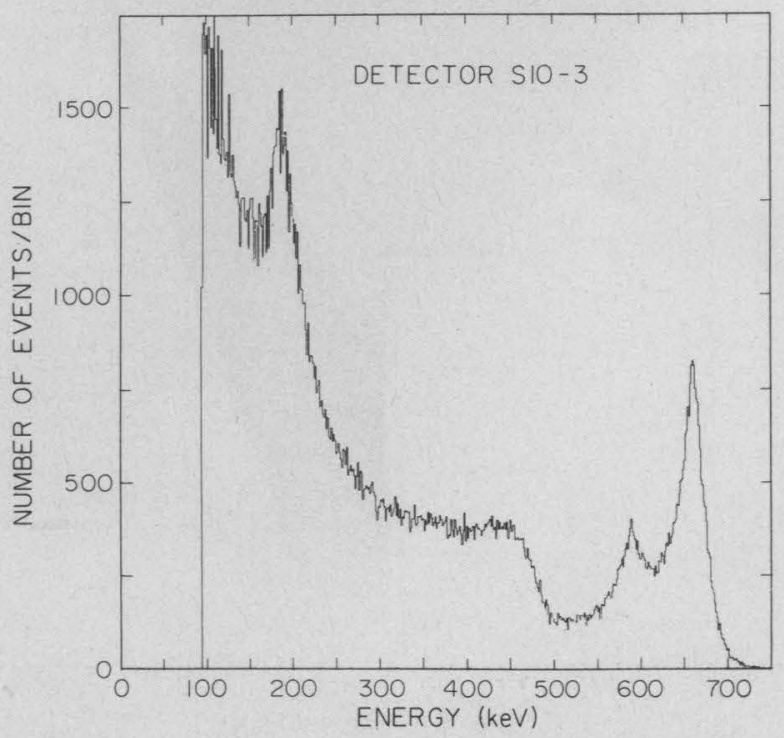

Figure 6c: Histogram of corrected energy loss. 
assumed hole trapping times. The full energy track is reasonably well bounded by the computed signal correlation for trapping times of $0.75 \mu \mathrm{s}$ and $1.5 \mu \mathrm{s}$. A range of trapping lengths would be a natural consequence of nonuniformities in hole trap density across the detector. The basic model thus seems to be successful in explaining the general features of the fast versus slow signal behavior for detector S10-3.

A contrasting case is indicated in Figure 7a which shows the fast versus slow event distribution for detector 56-25. The tracks in this case have a linear slope rather than the exponential curvature seen in Figure 6a for detector S10-3. The model is unable to explain this linear rather than exponential characteristic. The same linear slope is also present, but to a lesser degree in detector S8-13.

We have no firm explanation at this time for the linear character of the energy loss tracks observed in some detectors. Such behavior could be explained if there was a fixed asymmetry in the number of electron and hole carriers, with an excess of electron carriers. Physically, such a situation might arise in several ways. For instance, there might be an initial excess in the number of electron carriers produced if some of these carriers were freed from low level traps that do not produce a mobile hole carrier. Alternatively, nonuniform hole trapping (in particular, separated regions of low and high trap density) could produce an effective asymmetry in the carrier concentration. Non-uniform electric field intensities may also produce tracks which deviate from the simple model. More detailed studies are needed before a firm explanation can be put forward.

Because of the severe slope present in Figure $7 \mathrm{a}$, it is clear that the nominal energy resolution of detector $56-25$ is very poor. In fact, no photopeak or escape peak were discernible for this detector using the slow $6.4 \mu$ s analysis channel alone. However, Figure $7 \mathrm{~b}$ shows the estimated energy loss distribution above $500 \mathrm{keV}$ obtained by summing along the tracks in Figure $7 \mathrm{a}$. It can be seen that even for this detector, which has very poor intrinsic resolution, the two dimensional pulse processing yields a clear full energy peak and a separated escape peak.

\section{Summary}

The two dimensional pulse processing technique has two important applications. First, it can be used to significantly enhance the energy resolution of $\mathrm{HgI}_{2}$ detectors for low energy $\gamma$-rays without a compromise in efficiency. Second, the

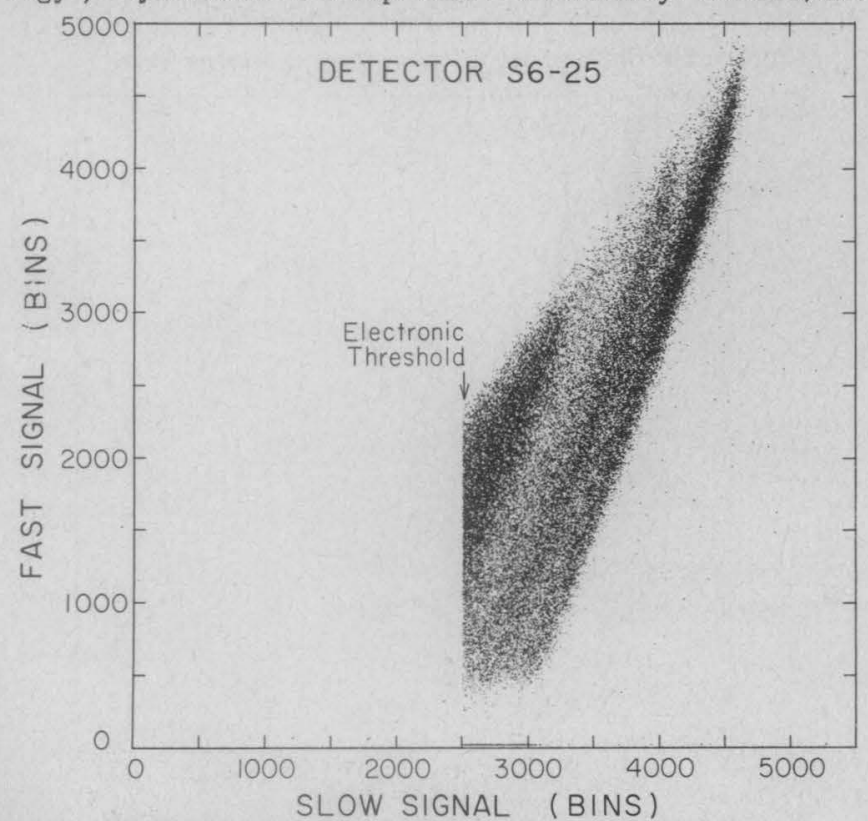

Figure 7a: Fast versus slow signal distribution. technique provides a useful tool for probing the nature of the charge carrier transport and trapping in solid state detectors. The method is not limited to $\mathrm{HgI}_{2}$ detectors, but rather should also be applicable to other detectors such as CdTe which have unequal hole and electron mobilities.

Further refinements are certainly possible. Mapping of the detector with a collimated beam of $\gamma$-rays would be useful in identifying spatial variations in detector response. More advanced electronic processing could also be implemented. In particular the use of current rather than voltage amplifiers in the fast processor would be useful, as would signal samples at several times during the current pulse. Ultimately, a waveform digitizer with sampling times on the order of $10 \mathrm{~ns}$ would completely characterize the current pulse.

\section{Acknowledgements}

This work was supported in part by the Jet Propulsion Laboratory Director's Discretionary Fund, NASA grant NGR 05002-160, and by U.S. Department of Energy Contract DEACOB-83NV10282. We thank the High Energy Astrophysics Group at the Jet Propulsion Laboratory under the direction of Alan Jacobson for their contributions to the project. The support and contributions of Profs. Stone and Vogt and W. Althouse at the California Institute of Technology are gratefully acknowledged.

\section{References}

[1] R. C. Whited, and M. M. Schieber, "Cadmium Telluride and Mercuric Iodide Gamma Radiation Detectors," Nucl. Inst. and Meth., vol. 162, pp. 113-123, 1979.

[2] A. J. Dabrowski, J. S. Iwanczyk, J. B. Barton, G. C. Huth, R. C. Whited, C. Ortale, T. E. Economou, and A. L. Turkevich, "Performance of Room Temperature Mercuric Iodide $\left(\mathrm{HgI}_{2}\right)$ Detectors in the Ultralow-Energy X-Ray Region," IEEE Trans. Nucl. Sci., vol. 28, no. 1, p. 536, 1981.

[3] L. T. Jones and P. M. Woollam, "Resolution Improvement in CdTe Gamma Detectors Using Pulse Shape Discrimination," Nucl. Inst. and Meth., vol. 124, pp. 591-595, 1975.

[4] R. C. Whited, M. M. Schieber, and P. T. Randtke, "Room temperature Gamma Spectroscopy of $\mathrm{Cs}^{137}$ using $\mathrm{HgI}_{2}$ Detectors," J. Appl. Phy., vol. 47, p. 2230, 1976.

[5] R. Kurz, "A Novel Pulse Processing System for $\mathrm{HgI}_{2}$ Detectors," Nucl. Inst. and Meth., vol. 150, pp. 91-95, 1978.

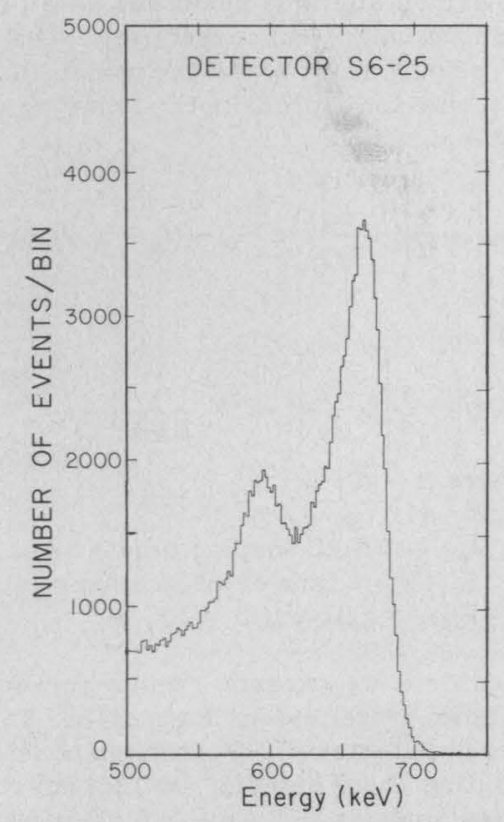

Figure 7b: Histogram of corrected energy loss. 